\title{
Tensioned Fabric Structures with Surface in the Form of Chen-Gackstatter
}

\author{
Hooi Min Yee, and Mohd Nasir Abdul Hadi \\ Universiti Teknologi MARA, 13500 Permatang Pauh, Pulau Pinang, Malaysia
}

\begin{abstract}
Form-finding has to be carried out for tensioned fabric structure in order to determine the initial equilibrium shape under prescribed support condition and prestress pattern. Tensioned fabric structures are normally designed to be in the form of equal tensioned surface. Tensioned fabric structure is highly suited to be used for realizing surfaces of complex or new forms. However, research study on a new form as a tensioned fabric structure has not attracted much attention. Another source of inspiration minimal surface which could be adopted as form for tensioned fabric structure is very crucial. The aim of this study is to propose initial equilibrium shape of tensioned fabric structures in the form of Chen-Gackstatter. Computational form-finding using nonlinear analysis method is used to determine the Chen-Gackstatter form of uniformly stressed surfaces. A tensioned fabric structure must curve equally in opposite directions to give the resulting surface a three dimensional stability. In an anticlastic doubly curved surface, the sum of all positive and all negative curvatures is zero. This study provides an alternative choice for structural designer to consider the Chen-Gackstatter applied in tensioned fabric structures. The results on factors affecting initial equilibrium shape can serve as a reference for proper selection of surface parameter for achieving a structurally viable surface.
\end{abstract}

\section{Introduction}

Tensioned Fabric Structures (TFS) are the composition of tensioned fabric as structural members where the fabric patterns are joined together at seams. It is then tensioned through mechanicals means or cables to a rigid supporting system to provide roofing structure. Figure 1 shows the example of tensioned fabric structure.

TFS include a wide variety of systems that are distinguished by their reliance upon tensile only members to support load. TFS have been employed throughout recorded history as in rope bridges and tents. However, large permanent tension structures were generally a 19th century development in bridges and a 20th century development in buildings. Tensioned fabric structure is the best alternative to cover area with low cost. One of the greatest TFS benefits is strength to weight ratio.

According to [1], TFS are durable structures and are good in insulation, light transmission and fire protection. All of these properties will be depending on the types of coating material used such as polyvinylchloride (PVC), polytetrafluoroethylene (PTFE) and ethylenetetrafluorethylene (ETFE). In addition, the membrane of TFS is made out of fibres. Those fibres are commonly made from nylon, polyester, glass and aramids.

TFS are normally designed to be in the form of equal tensioned surface. Minimal surface such as classical minimal surfaces, Costa and Möbius strip or their variation have been studied as possible choice of surface form for TFS by [2]-[11].
Tensioned fabric structure is highly suited to be used for realizing surfaces of new forms. However, none of the new examples mentioned present any results on the Chen-Gackstatter as load carrying members. Understanding of the possible Chen-Gackstatter initial equilibrium shapes to be obtained will provide alternative shapes for designers to consider. Form-finding using nonlinear analysis method can be used for both the initial equilibrium problem and load analysis for a new form of Chen-Gackstatter. This study is aimed at proposing a Chen-Gackstatter for structural designer to consider the Chen-Gackstatter applied in TFS.

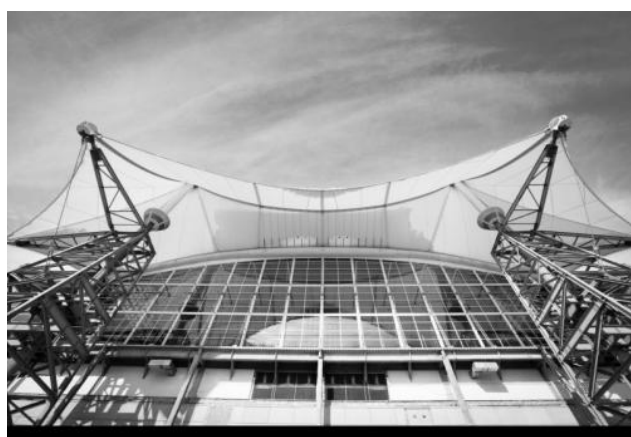

Figure 1. Denver International Airport (Redevelopment), 2014

\section{Generation of chen-gackstatter}

The name of Chen-Gackstatter is the name of two mathematicians Chi Cheng Chen and Fritz Gackstatter. 
This shape was found in 1982. According to [12] and [13] Chen-Gackstatter as appeared in the Figure 2 was the first complete orientable minimal surfaces of R3 and ChenGackstatter is derived from Enneper's minimal surface. It forms a double-indexed collection $[\mathrm{M}\rangle_{-}(\mathrm{i} j)$, where $\mathrm{i}$ $\geqslant 0$ and $j \geqslant 1$. The addition of $i$ makes the surface has topological genus equals to $i$.

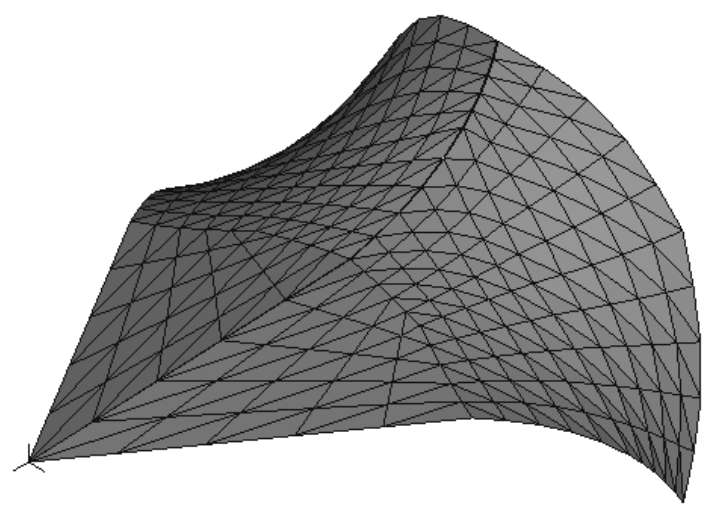

Figure 2. Chen-gackstatter minimal surface.

Figure 2 shows a model of Chen-Gackstatter. In this study, the XYZ coordinate of Chen-Gackstatter has been generated from Eq. (1). The software [14] has been used in this study.

$$
\begin{gathered}
X=\frac{4 A^{2}}{3 r^{3}} \cos (3 \theta) \\
Y=-\frac{4 A^{2}}{3 r^{3}} \sin (3 \theta) \\
Z=\frac{2 A}{r^{2}} \cos (2 \theta) \\
A=\sqrt{\frac{3 \pi}{2 g_{2}}} \\
g_{2}=60 \sum_{m, n=-\infty}^{\infty} \frac{1}{(m+n i)^{4}}
\end{gathered}
$$

$\mathrm{i}=$ imaginary unit

$\mathrm{r}=$ small positive constant

$$
0 \leqslant \theta \leqslant 2
$$

$\mathrm{g}_{2}=$ real and positive

\section{Form-finding using nonlinear analysis method}

The principle of proposed nonlinear analysis method by [2] is based on the large displacement finite element formulation used for analysis of structural behaviour under external loads. Since the method can be used for both the initial equilibrium problem and load analysis, the approach using nonlinear analysis is quite common. The basic equation used is expressed as follows:

$$
\left({ }_{0}^{t} \boldsymbol{K}_{L}+{ }_{0}^{t} \boldsymbol{K}_{G}\right) \boldsymbol{u}={ }^{t+\Delta t} \boldsymbol{F}-{ }_{0}^{t} \boldsymbol{f}
$$

where ${ }_{0}^{t} \boldsymbol{K}_{L}$ is linear strain incremental stiffness matrix, ${ }_{0}^{t} \boldsymbol{K}_{G}$ is nonlinear strain incremental stiffness matrix, ${ }_{0}^{t} \boldsymbol{f}$ is vector internal forces, ${ }^{\mathrm{t}+\Delta \mathrm{t}} \boldsymbol{F}$ is load vector and $\boldsymbol{u}$ is vector of increment in displacement.

Nonlinear finite element analysis procedures for stress analysis of TFS have been used as basis for form-finding analysis in this study. As a first shape for the start of form-finding analysis procedure adopted in this study, initial guess shape is needed. The software [14] has been used for the purpose of model generation. For the generation of such initial guess shape, anticlastic feature is incorporated into the model in order to produce a better initial guess shape. Such anticlastic feature has been incorporated by means of specification of selected sag, $\Delta$, relative to two suitably chosen points on the fixed boundary with span, $L$. Using this $\Delta / L$ sag ratio, geometry for approximates the surface of tensioned fabric structure is then generated. Such geometry is then meshed to produce initial guess shape with anticlastic feature.

The proposed computational strategy involves two stages of analysis in one cycle. The first stage (denoted as SF1) is an analysis which starts with an initial assumed shape in order to obtain an updated shape for initial equilibrium surface. This is then followed by the second stage of analysis (denoted as SS1) aimed at checking the convergence of updated shape obtained at the end of stage SF1. During stage SF1, elastic modulus E with very small values, are used. Warp and fill stresses, $\sigma_{\mathrm{W}}$ and $\sigma_{\mathrm{F}}$ are kept constant. In the second stage SS1, the actual tensioned fabric properties values are used. Resulting warp and fill stresses are checked at the end of the analysis against prescribed stresses. Iterative calculation has to be carried out in order to achieve convergence. The resultant shape at the end of iterative step $\mathrm{n}(\mathrm{SSn})$ is considered to be in the state of initial equilibrium under the prescribed warp and fill stresses and boundary condition if difference between the obtained and the prescribed tensioned fabric stresses relative to the prescribed stress satisfied the specified criteria. Such checking of difference in the obtained and prescribed stresses has been presented in the form of total stress deviation in warp and fill direction versus stress analysis stage.

In this study, three variable $r=0.3, r=0.6$ and $r=0.9$ of Chen-Gackstatter has been studied. This surface has been generated from Eq. (1) to create TFS model of ChenGackstatter. The number of nodes and triangular elements are 291 and 500, respectively for Chen-Gackstatter. The member pretension in warp and fill direction, denoted as $\sigma_{\mathrm{W}}$ and $\sigma_{\mathrm{F}}$ respectively, is $2000 \mathrm{~N} / \mathrm{m}$. The shear stress is zero. Young's Modulus-Membrane, E.t, is $800000 \mathrm{~N} / \mathrm{m}$, Shear modulus, Gt is $23529.41176 \mathrm{~N} / \mathrm{m}$ and Poisson' s Ratio, $\mathrm{v}_{\mathrm{xy}}=\mathrm{v}_{\mathrm{yx}}=0.7$. In this study, form-finding using 
nonlinear analysis method proposed by [2] in ChenGackstatter has been carried out.

\section{Result and discussion}

\subsection{Chen-Gackstatter, $r=0.3$}
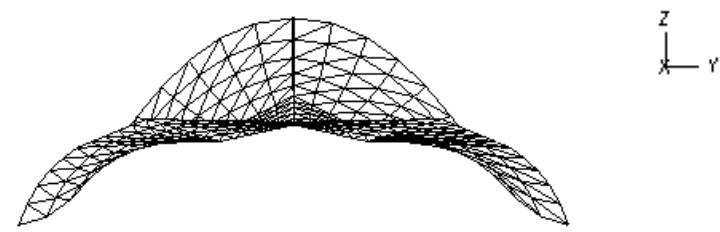

Front view

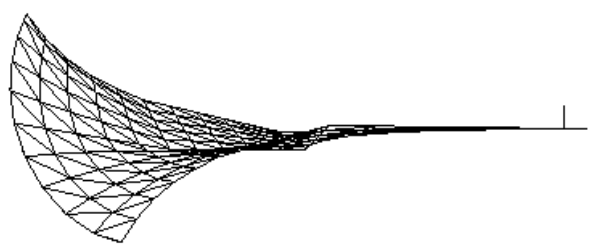

Side view

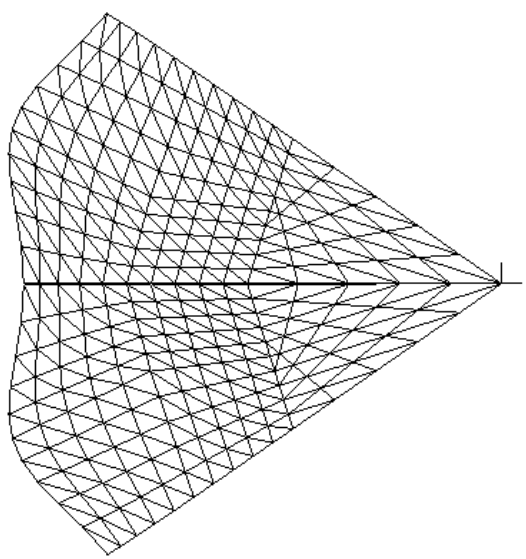

Top view

Figure 3. Initial GUESS shape of chen-gackstatter, $r=0.3$.

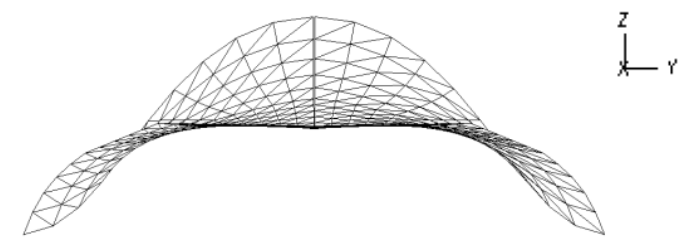

Front view

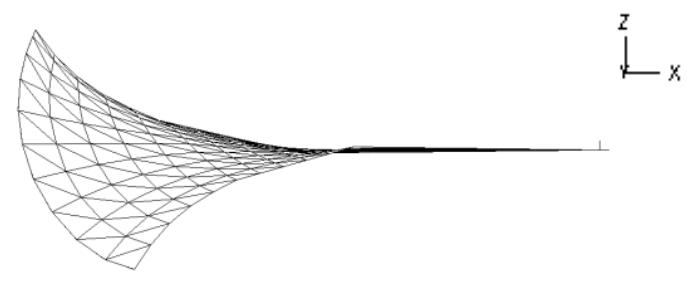

Side view

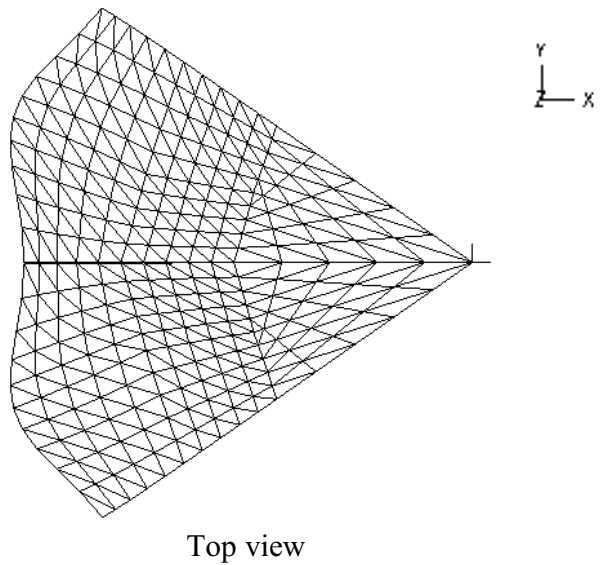

Figure 4. Converged shape of chen-gackstatter, $r=0.3$.

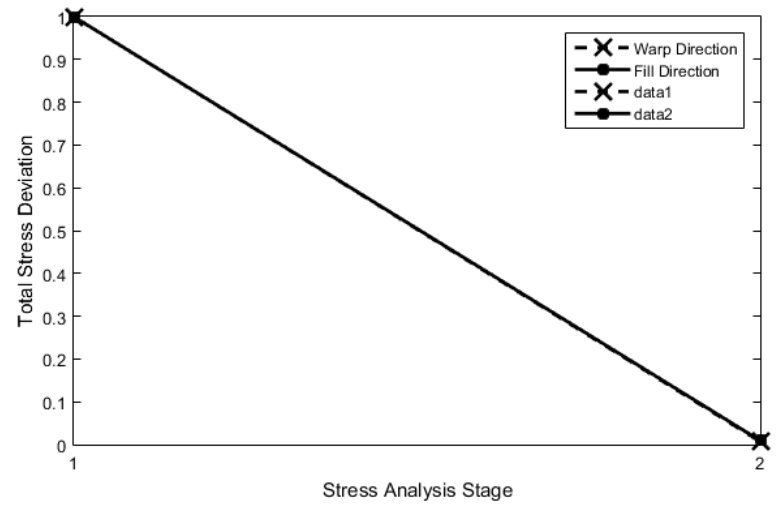

Figure 5. Convergent Curve of Chen-Gackstatter, $r=0.3$.

Figure 3 shows the initial guess shape of ChenGackstatter, $r=0.3$. Figure 4 shows the converged shape of the Chen-Gackstatter, $\mathrm{r}=0.3$ after form-finding. Figure 5 also shows the convergent curve of the ChenGackstatter. From this result, the warp and fill direction for Chen-Gackstatter of $\mathrm{r}=0.3$ is 0.006788 and 0.009175 . The convergent curve shows that the total warp and fill stress deviation of Chen-Gackstatter, $r=0.3<0.01$.

\subsection{Chen-gackstatter, $r=0.6$}

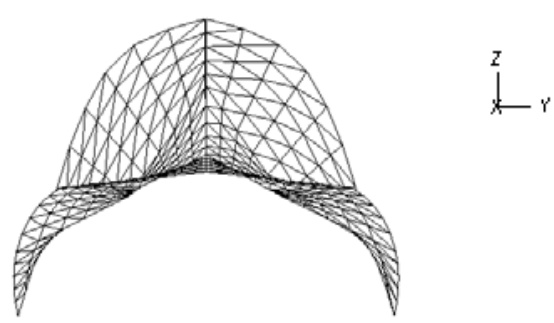

Front view

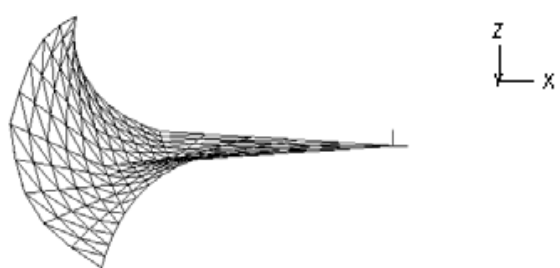

Side view 


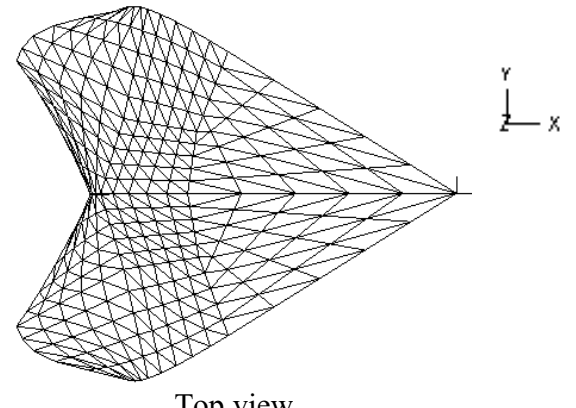

Top view

Figure 6. Initial guess shape of chen-gackstatter, $r=0.6$.

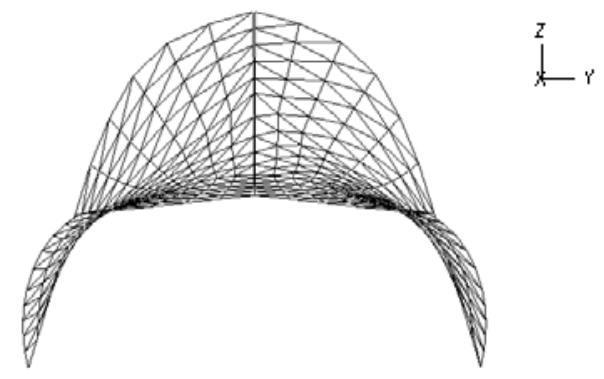

Front view

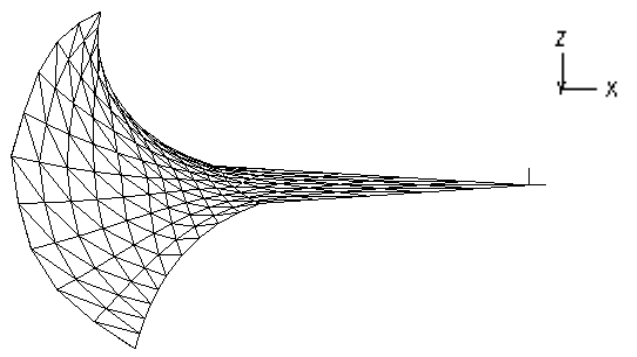

Side view
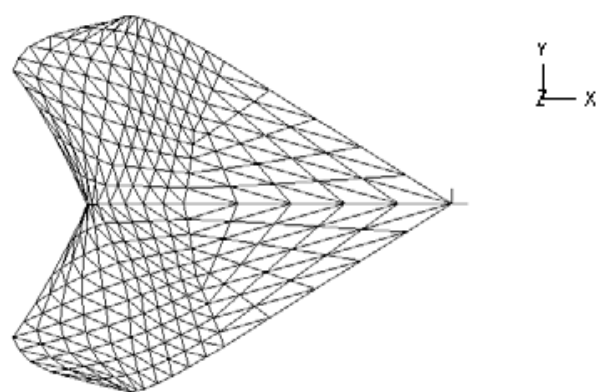

Top view

Figure 7. Converged shape of chen-gackstatter, $r=0.6$.

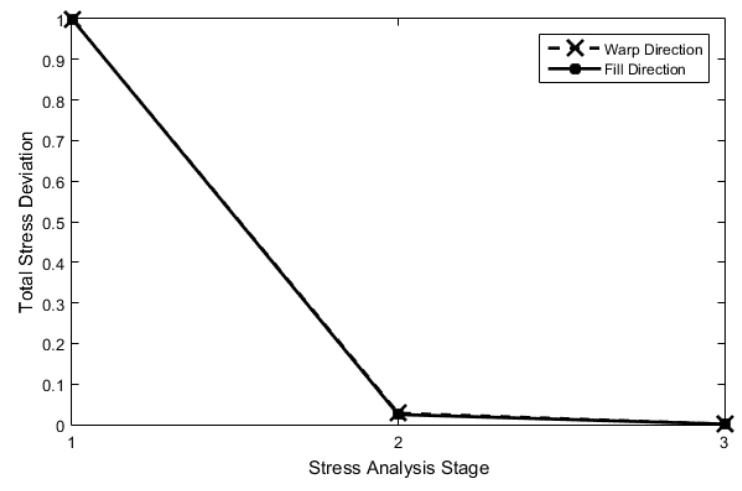

Figure 8. Convergent curve of chen-gackstatter, $r=0.6$
Figure 6 shows the initial guess shape of ChenGackstatter, $r=0.6$. Figure 7 shows the converged shape of the Chen-Gackstatter, $\mathrm{r}=0.6$ after form-finding. Figure 8 also shows the convergent curve of the ChenGackstatter. The warp and fill direction for ChenGackstatter of $\mathrm{r}=0.6$ is 0.001374 and 0.001211 . The convergent curve shows that the total warp and fill stress deviation of Chen-Gackstatter, $\mathrm{r}=0.6<0.01$.

\subsection{Chen-gackstatter, $r=0.9$}

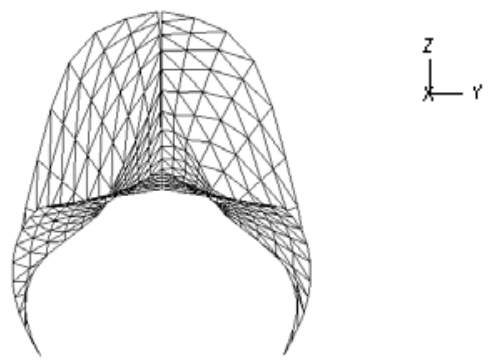

Front view
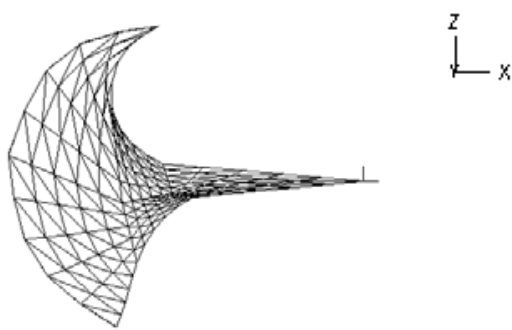

Side view

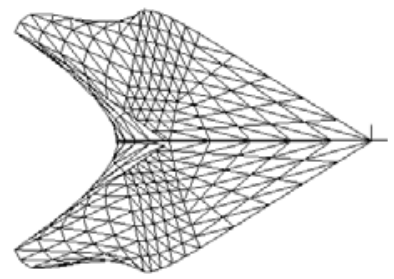

Top view

Figure 9. Initial guess shape of chen-gackstatter, $r=0.9$.

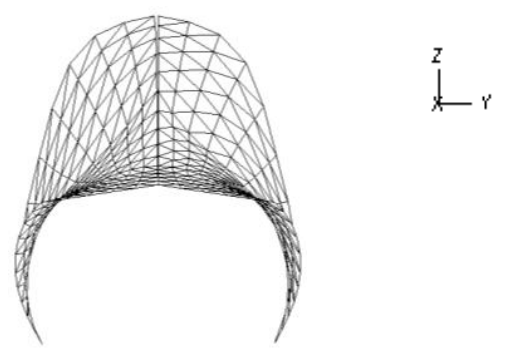

Front view
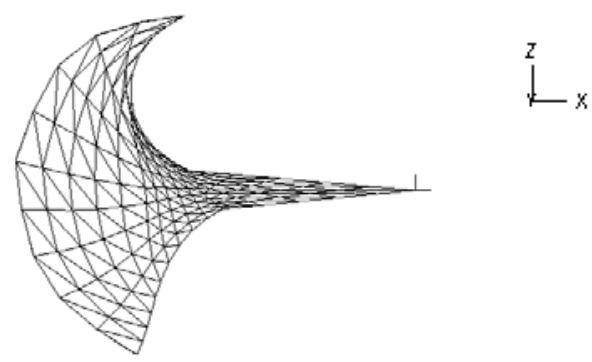

Side view 


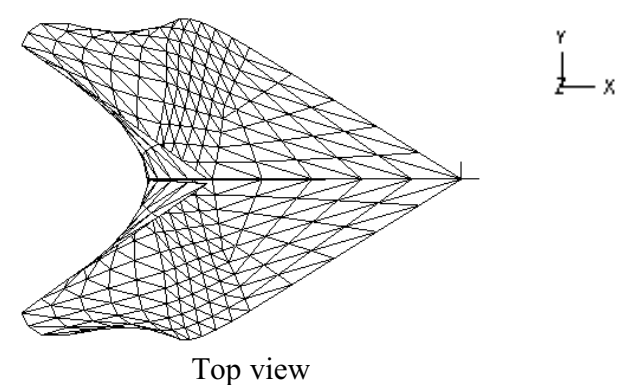

Figure 10. Converged shape of chen-gackstatter, $r=0.9$.

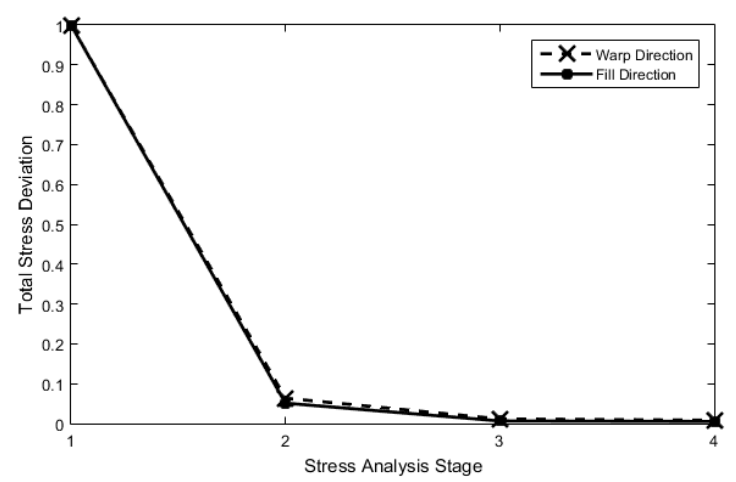

Figure 11. Convergent Curve of Chen-Gackstatter, $r=0.9$

Figure 9 shows the initial guess shape of ChenGackstatter, $r=0.9$. Figure 10 shows the converged shape of the Chen-Gackstatter, $\mathrm{r}=0.9$ after form-finding. Figure 11 shows the convergent curve of the Chen-Gackstatter. The warp and fill direction for Chen-Gackstatter of $r=0.9$ is 0.008255 and 0.005117 . The convergent curve shows that the total warp and fill stress deviation of ChenGackstatter, $r=0.9<0.01$.

\section{Conclusion}

Form Finding with Chen-Gackstatter with variable $r=0.3$, $r=0.6$ and $r=0.9$ has been carried out successfully using the procedure proposed by [2] which is based on nonlinear analysis method. The results from computational study show that TFS in the form of ChenGackstatter is a structurally viable surface form to be considered.

\section{References}

1. M.E. Son, The design and analysis of tension fabric structures, Massachusetts Institute of Technology, (2007)

2. H.M. Yee, A Computational Strategy for FormFinding of Tensioned Fabric Structure using Nonlinear Analysis Method, Ph.D Dissertation,
School of Civil Engineering, Universiti Sains Malaysia, Pulau Pinang, Malaysia (2011)

3. H.M. Yee, K.K. Choong, and J.Y. Kim, FormFinding Analysis of Tensioned Fabric Structures Using Nonlinear Analysis Method, Advanced Materials Research, 243, 249 1429-1434 (2011)

4. H.M. Yee and K.K. Choong, Form-Finding of Tensioned Fabric Structure in the Shape of Möbius Strip, Iranica Journal of Energy \& Environment, 4 (2013)

5. H.M. Yee, K.K. Choong, and J.Y. Kim, Experimental Form-Finding for Möbius Strip and Ennerper Minimal Surfaces Using Soap Film Models, International Journal of Engineering Science and Innovative Technology, 2, 328-335 (2013)

6. H.M. Yee, J.Y. Kim, and M.S. Mohd Noor, Tensioned Fabric Structures in Oval Form, Applied Mechanics and Materials, 405, 408 1008-1011 (2013)

7. M.S. Mohd Noor, H.M. Yee, K.K. Choong, and A.H. Haslinda, Tensioned Membrane Structures in the Form of Egg Shape, Applied Mechanics and Materials, 405, 408 989-992 (2013)

8. H.M. Yee and M.A. Samsudin, Development and Investigation of the Moebius Strip in Tensioned Membrane Structures, WSEAS Transactions on Environment and Development, 10, 145-149 (2014)

9. H.H Yee, K.K Choong, and M. A. Hadi, Sustainable Development of Tensioned Fabric Green Structure in the Form of Enneper, International Journal of Materials, Mechanics and Manufacturing, 3, 125-128 (2015)

10. H.M. Yee, A.H. Haslinda, and A.H. Nasir, Computer Investigation of Tensioned Fabric Structure in the Form of Enneper Minimal Surface, Applied Mechanics and Materials, 743-746 (2015)

11. H.M. Yee and M.A. Samsudin, Mathematical and Computational Analysis of Moebius Strip, International Journal of Mathematics and Computers in Simulation, 8, 197-201 (2014)

12. E.C. Thayer, Higher-Genus Chen-Gackstatter Surfaces and The Weierstrass Representation for Surfaces of Infinite Genus, Experimental Mathematics, 4, 19-39 (1995)

13. C.C. Chen and F. Gackstatter, Elliptische und hyperelliptische Funktionen und vollständige Minimalflächen vom Enneperschen Typ, Mathematische Annalen, 259, 359-369 (1982)

14. K..J. Bathe, ADINA system, ADINA R\&D Inc (2003) 